\title{
Análise das correlações existentes entre as medidas de aderência pneu-pavimento avaliadas em campo e em laboratório
}

\author{
Cláudia Azevedo Pereira ${ }^{1}$, Jorge Barbosa Soares², \\ Ivaldo Dário da Silva Pontes Filho ${ }^{3}$ e Verônica Teixeira Franco Castelo Branco ${ }^{4}$
}

\begin{abstract}
Resumo: O Brasil é um país que possui o seu sistema de transporte baseado principalmente no meio rodoviário, desta forma, a qualidade das estradas é primordial para o desenvolvimento do país. O presente estudo avalia as principais características de aderência pneu-pavimento de rodovias dos estados de Pernambuco e da Paraíba e verifica a possibilidade de se fazer previsões baseadas em simulações de laboratório. Para tal foram realizados os ensaios de Mancha de Areia, de Pêndulo Britânico e de Drenabilidade, e posteriormente foram também realizados os cálculos para a verificação do International Friction Index (IFI) de pontos selecionados em pavimentos das referidas rodovias. Em seguida, foram levantadas as características das misturas asfálticas utilizadas em campo sendo que duas delas foram simuladas em laboratório para verificação de indicadores de superfície relacionados à aderência pneu-pavimento. Por fim, foram analisadas duas misturas asfálticas concebidas de maneiras diferentes, uma com graduação contínua e outra descontínua, para verificar a possibilidade de ranqueamento entre elas no que diz respeito aos parâmetros que regem a aderência. Assim, obtem-se parâmetros que possibilitem uma previsão de comportamento em laboratório de misturas executadas em campo. DOI:10.4237/transportes. v20i4.598.
\end{abstract}

Palavras-chave: pavimento, segurança, aderência, misturas asfálticas.

\begin{abstract}
Brazil has its transportation system based mainly on roads, thus the quality of the roads is essential for the development of the country. This study evaluates the skid resistance characteristics in highways in the states of Pernambuco and Paraíba, verifying the possibility of making predictions based on laboratory simulations. For this purpose, the following tests were used: sand patch, British pendulum, and outflow meter and later the calculations were also performed to verify the International Friction Index (IFI) of selected points on the investigated roads. Then, two mixtures used in the field were reproduced in the laboratory and tested with simulated traffic to check their behavior with respect to skid resistance. Finally, two mixtures were designed, one discontinuous and one dense, in order to verify the possibility of ranking them according to their friction potential. With the results, can get parameters that allow a prediction in laboratory for the field behavior of these mixtures.
\end{abstract}

Keywords: pavement, safety, skid resistance, asphalt mixtures.

\section{INTRODUÇÃO}

Pode-se dizer que a matriz de transporte brasileira é predominantemente rodoviária, com esta modalidade correspondendo a cerca de $96,0 \%$ da matriz de transporte de passageiros e a $61,1 \%$ da matriz de transporte de cargas (CNT, 2011). Estudos feitos pela COPPEAD e pela Confederação Nacional do Transporte (CNT, 2007) mostram que, a participação do setor de transportes na economia brasileira adiciona cerca de $\mathrm{R} \$ 42$ bilhões ao PIB. Mesmo com tamanha importância, o setor ainda sofre com a falta de recursos, apesar dos investimentos realizados nos anos recentes. A disponibilidade de rodovias pavimentadas no Brasil é ainda pequena. Em 2010 eram cerca de 200 mil $\mathrm{km}$ pavimentados sobre um total de aproximadamente 1,7

${ }^{1}$ Cláudia Azevedo Pereira, Instituto Federal de Educação Ciência e Tecnologia de Goiás, Anápolis, GO, Brasil. (e-mail: claudia.azevedo@gmail.com).

2 Jorge Barbosa Soares, Departamento de Engenharia de Transportes, Universidade Federal do Ceará, Fortaleza, CE, Brasil. (e-mail: jsoares@det. ufc.br).

${ }^{3}$ Ivaldo Dário da Silva Pontes Filho, Departamento de Engenharia Civil, Universidade Federal de Pernambuco, Recife, PE, Brasil. (e-mail: ivaldo@ ufpe.br).

${ }^{4}$ Verônica Teixeira Franco Castelo Branco, Departamento de Engenharia de Transportes, Universidade Federal do Ceará, Fortaleza, CE, Brasil. (email: veronica@det.ufc.br).

Manuscrito recebido em 27/7/2012 e aprovado para publicação em 19/10/2012. Este artigo é parte de TRANSPORTES v. 20, n. 4, 2012. ISSN: 2237-1346 (online). DOI:10.4237/transportes.v20i4.598. milhão de km de rodovias. De acordo com CNT (2011), entre os anos de 2000 e 2010, foi investido em média, $0,2 \%$ do PIB anual em infraestrutura de transporte. Já em 2010, com os incentivos do Programa de Aceleração do Crescimento (PAC), o Brasil investiu 0,36\% do PIB anual em infraestrutura de transporte, um avanço se comparado à década anterior, mas muito aquém do investimento feito por países como China, Índia e Rússia, que investiram, respectivamente, $10 \%, 8 \%$ e $7 \%$ do seu PIB neste setor (CNT, 2011). A pesquisa CNT (2011) salienta ainda que, em 2010, foram perdidos $\mathrm{R} \$ 14,1$ bilhões em acidentes rodoviários nas vias federais policiadas. Este montante representa uma perda de $0,4 \%$ do PIB deste ano, ou seja, um valor além daquele investido em infraestrutura de transporte no mesmo ano. Desta forma, ainda existe muito a ser feito no país, pois a infraestrutura de transporte tem sido um gargalo para o desenvolvimento. Neste contexto, medidas que possam proporcionar um melhor conhecimento das práticas e materiais empregados em pavimentação são essenciais.

Um dos materiais mais empregados em revestimentos de pavimentos no Brasil é o Concreto Asfáltico (CA). Este é considerado um material composto de cimento asfáltico de petróleo, partículas finas, agregados miúdos e graúdos, além de vazios. Cada componente possui individualmente propriedades químicas e mecânicas (Yue et al., 1995). O comportamento da mistura é influenciado por vários fatores como as propriedades de cada componente, do conjunto de 
componentes, assim como o grau de compactação da mesma (Masad et al., 1999a apud Vasconcelos et al., 2005).

A estrutura interna da mistura asfáltica possui um importante papel na resistência do pavimento com relação à fadiga, ao trincamento térmico, dentre outros (Masad et al., 1999). Desta forma, têm sido desenvolvidos diversos estudos que visam quantificar parâmetros importantes sobre os agregados nas misturas, tais como: orientação do agregado, granulometria, forma, etc. Estas medidas são usadas para comparar a estrutura interna do CA preparado utilizando diferentes níveis de compactação, por exemplo, no Compactador Giratório Superpave (CGS) (Pereira, 2010). Contudo, no aspecto de aderência pneu-pavimento, ainda existem poucos estudos que contemplam as correlações existentes entre campo e laboratório. Desta forma, existem pavimentos que são concebidos sem a preocupação com este parâmetro, ocasionando uma falta de segurança nas rodovias.

O presente trabalho visa analisar as correlações entre campo e laboratório com relação aos ensaios de aderência pneu-pavimento. Para isto, foram realizados ensaios de Mancha de Areia, Pêndulo Britânico e uma avaliação utilizando a classificação IFI - International Friction Index - na versão de 1998 em pavimentos dos estados de Pernambuco e da Paraíba. Também foram realizados ensaios em laboratório com a reprodução de duas das misturas utilizadas em campo para verificar os seus desempenhos em estágio inicial e a possibilidade de realizar previsões em laboratório para situações ocorridas em campo. Por fim, foram realizados ensaios em laboratório para duas misturas com concepções granulométricas diferentes, uma contínua e outra descontínua, para verificação de um possível ranqueamento entre estas misturas quanto à aderência.

\section{REVISÃO BIBLIOGRÁFICA}

\subsection{Aderência Pneu-Pavimento}

O comportamento da superfície de uma rodovia, sob o aspecto da aderência, pode ser descrito por uma parcela de atrito e outra de textura do revestimento. Alguns autores afirmam que a textura possui uma importante ação no atrito a altas velocidades e em pistas molhadas, demonstrando haver correlação entre a textura do revestimento e os índices de derrapagem (Meurer, 2001 apud Ferreira, 2002). Uma microtextura adequada favorece o rompimento da película de água presente entre o pneu e a superfície das asperezas do pavimento, influenciando na parcela de atrito. Já a macrotextura é importante por favorecer a drenagem d'água encontrada na interface pneu-pavimento (Brosseaud, 2006). De maneira geral, pode-se dizer que, a microtextura corresponde a aspereza ou a rugosidade da superfície individual dos agregados que compõem a mistura asfáltica ou o Concreto de Cimento Portland (CCP) e não pode ser observada a olho nu. A macrotextura corresponde ao tamanho do agregado, à faixa granulométrica, aos vazios existentes na mistura, e a configuração geométrica individual do agregado (Lay, 1998). Desta forma, a superfície resultante depende das características de micro e macrotextura conjuntamente (Pereira, 2010). As combinações destas características podem resultar em quatro tipos de textura: (i) rugosa e aberta; (ii) rugosa e fechada; (iii) polida e aberta; e (iv) polida e fechada (Aps, 2006).

Com relação às medidas de aderência pneu-pavimento, existe o IFI (International Friction Index), este índice combina medidas de atrito e de textura, e possibilita verificar a situação dos pavimentos perante estes parâmetros. Neste trabalho foram realizados os ensaios de pêndulo britânico, como medida de atrito e mancha de areia como medida de textura, além do ensaio de drenabilidade. Posteriormente, foi feito o cálculo do IFI.

O pêndulo britânico é uma medida indireta do coeficiente de atrito realizada através de um equipamento associado a um método padronizado pela ASTM E303-98. Este possui uma haste metálica que pode girar em torno de uma rótula, em cuja extremidade oposta encontra-se uma sapata com base de borracha, que é lançada em queda livre em direção ao revestimento molhado, com o objetivo de medir a perda de energia por atrito da sapata de borracha do pêndulo quando esta desliza sobre a superfície do pavimento. $\mathrm{O}$ valor é expresso em BPN (British Number Pendulum) ou SRT (Skid Resistent Test) (Pereira et al., 2011).

A mancha de areia é uma medida indireta de textura que serve para determinar a média da profundidade da altura da mancha de areia (HS) em superfícies de pavimentos, conforme especificação da ASTM E965-01. Este ensaio consiste em preencher os vazios da textura superficial do revestimento com um volume conhecido de $25.000 \mathrm{~mm}^{3}$, aproximadamente $150 \mathrm{~mm}^{3}$ de areia natural limpa e seca, uniforme, de grãos arredondados, que passa na peneira de abertura $0,3 \mathrm{~mm}$ e fica retida na peneira de abertura $0,15 \mathrm{~mm}$, ou com microesferas de vidro com as mesmas características granulométricas (Pereira, 2010).

O ensaio de drenabilidade consiste em avaliar a capacidade de escoamento da água superficial por entre os canais dos vazios da textura superficial e, desta forma, auxiliar na caracterização da macrotextura do revestimento. O equipamento utilizado é o drenômetro que segue as dimensões do Institute for Highway, Railroads and Rock Engineering (ISETH) (Pereira, 2010). O ensaio consiste em posicionar o drenômetro sobre a superfície do pavimento, previamente molhado, e enchê-lo com água. Esta água será drenada pelos canais existentes na superfície que a borracha do orifício do drenômetro não vedar. Mede-se, então, o tempo necessário para a água ser escoada, na pressão atmosférica de um volume prédeterminado (Ferreira, 2002).

O último passo refere-se ao cálculo do IFI, que pode ser obtido através da combinação de dois tipos de medidas, uma de atrito (FR) e outra de textura (Tx), homologadas ou homologáveis a partir de critérios estabelecidos em experimento internacional (ASTM E 1960-98). Em 2007, esta norma apresentou algumas alterações sendo denominada ASTM E 1960-07. Os princípios e as equações continuaram os mesmos da versão anterior. A alteração foi em relação aos valores das constantes dos equipamentos utilizados nestas citadas equações. A partir desta versão, estas constantes devem ser obtidas por meio de calibração do equipamento escolhido para medir textura e atrito, como os equipamentos CT-Meter (Circular Texture Meter) e DFTester (Dynamic Friction Tester). Entretanto, no Brasil a existência destes equipamentos é recente.

As análises de aderência pneu-pavimento geralmente são feitas somente em campo, não existindo, ainda, parâmetros de laboratório que possam predizer o comportamento da 
mistura. Neste sentido, Nunes et al. (2008) procuraram analisar a macrotextura do BBTM 0/6 (Béton Bitumineux Trés Mince) com amostras retiradas de campo e preparadas em laboratório e obtiveram alguns indicadores de que as misturas podem ser reproduzidas em laboratório de forma satisfatória. Outros aspectos que vêm sido desenvolvidos pelos pesquisadores dizem respeito à microtextura através da realização de ensaios específicos para a caracterização das propriedades de agregados.

Existem propriedades tais como a forma, a textura e a angularidade dos agregados que são de grande importância no desempenho do pavimento. Os métodos atuais utilizados para medir estas características possuem várias limitações: trabalhosos, subjetivos, não possuem relação direta com o desempenho e limitados com relação à separação dos resultados de angularidade e de textura. Vários estudos mostram que agregados que exibem textura rugosa não necessariamente possuem alta angularidade, especialmente em se tratando de agregados graúdos. Desta forma, é importante desenvolver métodos capazes de quantificar cada propriedade do agregado separadamente (Masad et al., 2005).

Outra medida importante para a pavimentação, em especial para a questão da aderência pneu-pavimento, é a resistência ao polimento do agregado. Esta afeta a microtextura do pavimento e, consequentemente, o atrito entre o pneu e o pavimento. Este parâmetro começou a ser melhor estudado recentemente com a utilização das características microscópicas das misturas asfálticas. Mahmoud (2005) salienta novos métodos de avaliação da resistência ao polimento dos agregados, sendo um deles através da utilização do AIMS (Aggregate Imaging Measurement System). De acordo com Bessa et al. (2011), com o AIMS pode-se obter cinco diferentes propriedades dos agregados: forma 2D, angularidade, textura, esfericidade e lamelaridade. Além disto, este equipamento também é capaz de fornecer a análise da textura superficial de corpos-de-prova de misturas asfálticas.

Além destas propriedades, deve-se atentar para as características de adesividade do agregado pois com a película de filme que cobre o mesmo, este fica mais protegido do desgaste sofrido pelo tráfego. Ao mesmo tempo, esta película cobre a microtextura do agregado ocasionando uma alteração nas características de atrito em relação ao seu estágio inicial de desgaste. Esta propriedade pode variar de acordo com a composição mineral, a aspereza da superfície, a forma do agregado e de suas arestas, a presença de umidade e as impurezas na superfície e a porosidade (Pereira et al., 2011).

Também é importante verificar o desempenho das diversas composições das misturas, e como estas se comportam ao longo do tempo sob a ação do tráfego. Existem vários métodos de melhoramento da textura e da drenagem superficial do revestimento. Como exemplo pode-se citar o grooving, a lama asfáltica e o microrrevestimento, ou misturas asfálticas com características específicas como a Camada Porosa de Atrito (CPA), o Stone Matrix Asphalt (SMA) e o Béton Bitumineux Très Mince (BBTM). Uma previsão do comportamento quanto à aderência para esses tipos de misturas pode auxiliar a tomada de decisão quanto ao melhor revestimento a ser utilizado diante dos recursos disponíveis.

\section{MÉTODO}

Para o atendimento do objetivo central da presente pesquisa, foi estabelecida uma programação de ensaios de campo em rodovias dos estados de Pernambuco e da Paraíba. A programação envolveu:

- Levantamento dos pontos para a realização dos ensaios em campo através das estatísticas de acidentes da Polícia Rodoviária Federal (PRF) e de técnicos do setor;

- Ensaios em campo: pêndulo britânico, mancha de areia e drenabilidade;

- Cálculo do IFI;

- Levantamento das misturas asfálticas utilizadas em campo;

- Simulação em laboratório de duas misturas utilizadas em campo; e

- Confecção de duas misturas de concepções diferentes, uma de graduação contínua e outra descontínua, para verificação da possibilidade de ranqueamento entre elas.

\subsection{Local de Estudo}

Foram analisados os trechos de maior incidência de acidentes e incidentes nos estados de Pernambuco e da Paraíba. Em Pernambuco, os dados foram obtidos através de estatísticas da $11^{\mathrm{a}}$ Superintendência da PRF, sediada em Recife. $\mathrm{Na}$ Paraíba, a escolha foi tomada com base em relatos de técnicos do Departamento de Estrada de Rodagem (DER) da Paraíba e da Universidade Federal de Campina Grande (UFCG), além das observações dos policiais rodoviários federais.

\subsection{Ensaios e procedimentos utilizados na pista}

Foram realizados os ensaios listados a seguir:

- Pêndulo britânico: o ensaio baseou-se no método ASTM E 303-98 e foi adotada a classificação apresentada na Tabela 1.

- Mancha de areia: o ensaio baseou-se na ASTM E 965-01. O Manual de Reabilitação de Pavimentos Asfálticos do DNER (1998) apresenta faixas de valores para a macrotextura entre 0,6 e $1,2 \mathrm{~mm}$. Entretanto, para tornar compatível com o número de faixas utilizadas no ensaio com o pêndulo britânico, foram incorporadas mais duas faixas na classificação da mancha de areia (Aps, 2006). Os novos limites adotados no estudo estão expressos na Tabela 1.

- Drenabilidade: foi utilizado o drenômetro, que consiste em um cilindro de acrílico transparente. No terço médio da altura do cilindro existem duas linhas que delimitam um volume conhecido de água (0,73litro), cujo tempo de escoamento é registrado permitindo conhecer a capacidade de escoamento da água superficial. Foi utilizado o drenômetro que segue as dimensões do Institute for Highway, Railroads and Rock Engineering (ISETH).

Posteriormente, prosseguiu-se com os cálculos de IFI de acordo com a versão da norma de 1998. As faixas de classificação de IFI, propostas por APS (2006) e consideradas nesta pesquisa, são apresentadas também na Tabela 1. A referida autora sugere ainda a exigência dos valores mínimos de 0,15 e 0,22 para pavimentos em uso e novos, respectivamente. 
Tabela 1. Classificação a partir dos resultados do pêndulo britânico (APS, 2006)

\begin{tabular}{|c|c|c|c|c|c|}
\hline Pêndulo Britânico & & Mancha de Areia & & IFI & \\
\hline Classificação (Legenda) & Limites $B P N$ & Classificação & Limites HS (mm) & Classificação & Limites IFI \\
\hline Perigosa $(\mathrm{P})$ & $\mathrm{BPN}<25$ & Muito Fina (MF) & $\mathrm{HS}<0,20$ & Péssimo & IFI $<0,05$ \\
\hline Muito Lisa (ML) & $25 \leq \mathrm{BPN}<31$ & Fina $(\mathrm{F})$ & $0,20<\mathrm{HS}<0,40$ & Muito Ruim & $0,06 \leq \mathrm{IFI}<0,08$ \\
\hline Lisa (L) & $32<\mathrm{BPN}<39$ & $\begin{array}{l}\text { Medianamente } \\
\text { Fina }(\mathrm{MeF})\end{array}$ & $0,40<\mathrm{HS}<0,60$ & Ruim & $0,09 \leq \mathrm{IFI}<0,11$ \\
\hline Insuficientemente Rugosa (IR) & $40<\mathrm{BPN}<46$ & Média (M) & $0,60<\mathrm{HS}<0,80$ & Regular & $0,12 \leq$ IFI $<0,14$ \\
\hline Medianamente Rugosa (MR) & $47<\mathrm{BPN}<54$ & $\begin{array}{l}\text { Medianamente } \\
\text { Grossa }(\mathrm{MeG})\end{array}$ & $0,80<\mathrm{HS}<1,00$ & Bom & $0,15 \leq$ IFI $<0,21$ \\
\hline Rugosa (R) & $55<\mathrm{BPN}<75$ & Grossa (G) & $1,00<\mathrm{HS}<1,20$ & Muito Bom & $0,22 \leq$ IFI $<0,35$ \\
\hline Muito Rugosa (MuR) & $\mathrm{BPN}>75$ & Muito Grossa (MG) & $\mathrm{HS}>1,20$ & Ótimo & IFI $>0,35$ \\
\hline
\end{tabular}

\subsection{Ensaios e procedimentos utilizados em campo}

Após a realização dos ensaios em campo, foram levantadas informações sobre os traços das misturas avaliadas na pista. Por questões práticas, as misturas receberam a nomenclatura apresentada na Tabela 2 e as composições granulométricas destas misturas podem ser observadas na Figura 1. A ideia foi analisar a influência que a composição da mistura exerce sobre a aderência pneu-pavimento.

Posteriormente, tentou-se simular em laboratório as situações observadas em campo. Para isto, foi adquirido agregado da região onde foram executadas as obras para que suas características mineralógicas e físicas fossem as mais próximas possíveis daquelas encontradas para o agregado utilizado em campo. O ligante utilizado também foi o mais próximo, dentro das possibilidades, daquele utilizado nas obras. Contudo, tanto o ligante utilizado na pista como aquele utilizado na simulação em laboratório foi o CAP 50/70, procedente da Lubnor (Lubrificantes e Derivados de Petróleo do Nordeste/Petrobras) no Ceará.

Dois tipos de misturas utilizadas em campo foram confeccionadas em laboratório, uma com Tamanho Máximo Nominal (TMN) de $9,5 \mathrm{~mm}$ e outra com TMN de $12,5 \mathrm{~mm}$. As misturas confeccionadas foram denominadas Mistura D e Mistura F. Em seguida, estas misturas foram moldadas em uma mesa compactadora do tipo LCPC seguindo as di-

Tabela 2. Especificação dos tipos de superfícies ensaiadas (PEREIRA, 2010)

\begin{tabular}{|c|c|c|c|c|}
\hline Rodovia & $\operatorname{Trecho}(\mathrm{km})$ & Tipo de Superfície & $\begin{array}{l}\text { Nomenclatura } \\
\text { adotada da mistura }\end{array}$ & $\begin{array}{l}\text { Ano de } \\
\text { intervenção }\end{array}$ \\
\hline & 33 & Concreto com capa de CA & $\mathrm{S} / \mathrm{I}(\mathrm{A})$ & 1973 \\
\hline & 49 & $\mathrm{CA}$ & $\mathrm{S} / \mathrm{I}(\mathrm{B})$ & 2001 \\
\hline & 67 e 73 & Concreto com capa de CA & $\mathrm{S} / \mathrm{I}(\mathrm{C})$ & 2001 \\
\hline & 83,94 e 98 & $\mathrm{CA}$ & Mistura A & 2007 \\
\hline & 114,132 e 145 & $\mathrm{CA}$ & Mistura B & 2001 \\
\hline \multirow[t]{5}{*}{ BR 101-PE } & 157 e 161 & $\mathrm{CA}$ & Mistura C & 2001 \\
\hline & 6 & $\mathrm{CCP}$ & $\mathrm{S} / \mathrm{I}(\mathrm{D})$ & 2002 \\
\hline & 7 e 8 & Concreto com capa de CA & $\mathrm{S} / \mathrm{I}(\mathrm{E})$ & 2002 \\
\hline & 9 e 15 & $\mathrm{CA}$ & Mistura D & 2002 \\
\hline & $75,90,91$ e 110 & $\mathrm{CCP}$ & $\mathrm{S} / \mathrm{I}(\mathrm{F})$ & 2002 \\
\hline \multirow[t]{4}{*}{ BR 232-PE } & 147,160 e 175 & TSD & $\mathrm{S} / \mathrm{I}(\mathrm{G})$ & 2007 \\
\hline & $138 \mathrm{AC}^{*}, \mathrm{C}^{*} \mathrm{e} \mathrm{DC}^{*}$ & $\mathrm{CA}$ & Mistura E & 2000 \\
\hline & $115 \mathrm{AC}^{*}, \mathrm{C}^{*} \mathrm{e} \mathrm{DC}^{*}$ & CA & Mistura F & 2000 \\
\hline & $67 \mathrm{AC}^{*}, \mathrm{C}^{*}$ e $\mathrm{DC}^{*}$ & $\mathrm{CA}$ & Mistura G & 2000 \\
\hline BR 230-PB & $49 \mathrm{AC}^{*}, \mathrm{C}^{*} \mathrm{e} \mathrm{DC}^{*}$ & $\mathrm{CA}$ & Mistura G & 2000 \\
\hline
\end{tabular}

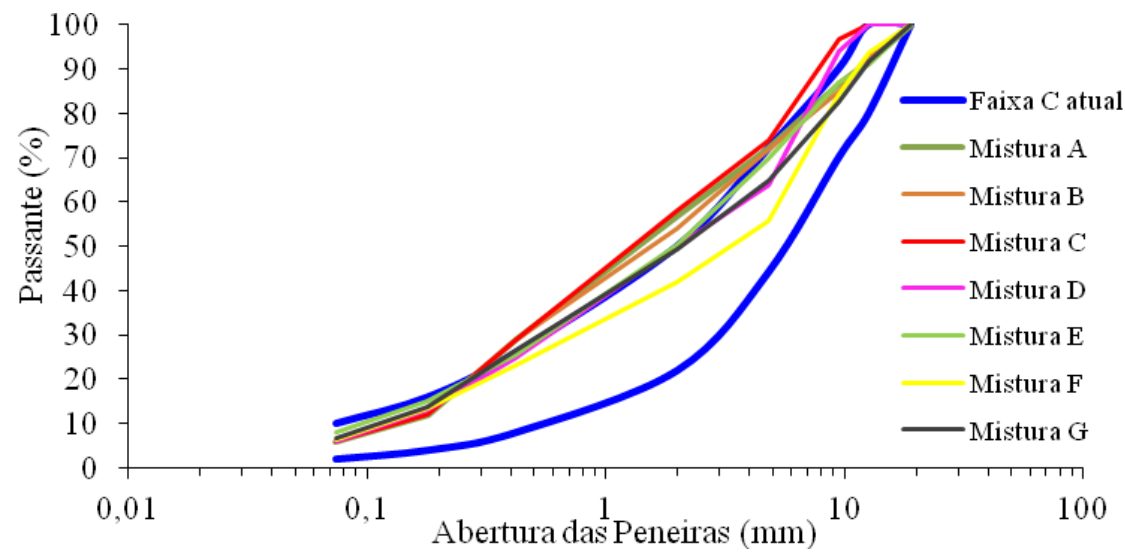

Figura 1. Granulometria das misturas encontradas em campo 
retrizes da norma francesa NF P 98-253-1 com algumas adaptações sugeridas pelo Laboratório de Tecnologia de Pavimentação (LTP) da Poli/USP (Pereira et al., 2011).

Após a compactação, a mistura foi colocada no simulador de tráfego do tipo LCPC e mediu-se a deformação na mesma nos ciclos referentes a 100, 300, 1.000, 3.000, 10.000 e 30.000 passagens. Simultaneamente à realização deste ensaio, foram realizados os ensaios de mancha de areia e de pêndulo britânico. $\mathrm{O}$ ensaio de mancha de areia foi realizado antes do início da simulação e nos ciclos de número $100,300,1.000,3.000,10.000$ e 30.000. Para que fosse possível realizar o ensaio na superfície da placa, este foi feito com metade do volume padrão de areia (Pereira et al., 2011).

O ensaio de pêndulo britânico foi realizado antes da simulação de tráfego e após o ciclo 10.000 , devido à dificuldade de se realizar o ensaio a cada ciclo. Também não foi possível realizar este ensaio aos 30.000 ciclos, pois com a formação da trilha de roda não foi possível a passagem da sapata do pêndulo de forma adequada para a realização do ensaio. Entretanto, como o importante é saber como ficaria o atrito inicial e em estágio avançado de passagem de tráfego, acredita-se que as medidas inicial e após 10.000 ciclos sejam suficientes para a realização da análise.

\subsection{Verificação das misturas com concepções diferentes}

Para a confecção das misturas realizou-se a caracterização de todos os materiais. Para os agregados foram utilizados os procedimentos convencionais preconizados pelo DNIT, além de alguns testes exigidos nas especificações Superpave. Dois tipos de misturas asfálticas foram investigadas: CA e SMA. As misturas foram dosadas em laboratório e sua caracterização foi realizada através de Corpos de Provas (CPs) compactados no Compactador Giratório Superpave (CGS). Por fim, foram moldadas placas na mesa compactadora do simulador de tráfego laboratorial, da Universidade Federal do Ceará (UFC) e realizados os ensaios de pêndulo britânico, mancha de areia e drenabilidade nestas placas. A composição granulométrica das misturas adotadas estão expressos na Figura 2.

\section{ANÁLISE DOS RESULTADOS}

\subsection{Comparação dos resultados entre as misturas de campo simuladas em laboratório}

Os valores encontrados em campo são apresentados por Pereira (2010). Observou-se, através da Tabela 3, que, em grande parte das estradas analisadas a situação é precária com relação à aderência pneu-pavimento. Cerca de $97 \%$ dos pontos precisam de algum tipo de intervenção, ou seja, precisam melhorar a micro ou a macrotextura. Quando são considerados os valores de IFI, estes foram satisfatórios, sendo que cerca de $50 \%$ dos pontos analisados apresentaram classificação entre boa e ótima de acordo com este parâmetro.

Analisando-se as Misturas D e F, que foram simuladas em laboratório, verificou-se que os resultados do ensaio de deformação permanente das misturas oriundas da BR 232 PE, designada Mistura D, e da BR 230 - PB, designada Mistura $\mathrm{F}$, apresentaram valores satisfatórios para este parâmetro, pois estão abaixo de $10 \%$ de deformação.

Com relação ao ensaio de mancha de areia realizado nas placas, pode-se verificar, através dos resultados apresentados na Tabela 4 e na Figura 3, que em nenhuma das misturas obteve-se um padrão de comportamento bem definido ao longo dos ciclos. Isto pode ser explicado devido ao fato da compactação em laboratório ser diferente da compactação em campo. Nunes et al. (2008) salientaram que a orientação dos agregados na superfície da mistura está fortemente ligada à compactação. No campo existe o processo de vibração, enquanto que no laboratório não. Com isto, acontece o tombamento dos agregados na primeira situação. Com o passar da roda na simulação, as partículas de agregado vão ficando cada vez mais salientes na superfície apresentando um falso ganho na medida de textura (Pereira, 2010).

Verificando as medidas de atrito apresentadas na Tabela 5 e na Figura 4, observou-se que elas apresentaram um padrão parecido com aquele encontrado em campo. Tanto em campo como em laboratório, a Mistura F apresentou-se superior no que diz respeito ao parâmetro de atrito quando comparada à Mistura $\mathrm{D}$, havendo apenas uma pequena variação na medida inicial, onde a Mistura D apresentou-se melhor. O desempenho encontrado com a evolução da simulação também foi o esperado, pois com a passagem da roda, houve descolamento da película de ligante e polimento dos agregados, favorecendo a diminuição da medida de

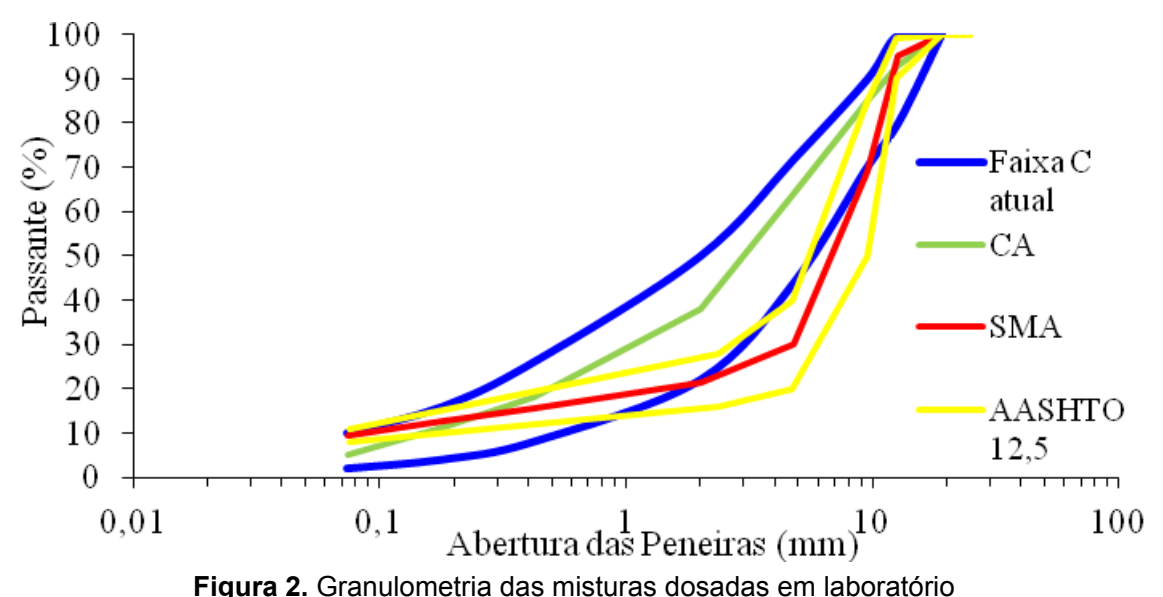


Tabela 3. Especificação dos tipos de superfícies ensaiadas (Pereira, 2010)

\begin{tabular}{|c|c|c|c|c|c|c|}
\hline \multirow[b]{2}{*}{ Rodovia } & \multirow[b]{2}{*}{$\operatorname{Trecho}(\mathrm{km})$} & \multirow{2}{*}{$\begin{array}{l}\text { Mancha de areia } \\
H S(\mathrm{~mm})\end{array}$} & \multirow{2}{*}{$\begin{array}{l}\text { Pêndulo britânico } \\
B P N\end{array}$} & \multicolumn{2}{|l|}{ IFI } & \multirow[b]{2}{*}{ Drenabilidade $(l / s)$} \\
\hline & & & & $S p$ & $F 60$ & \\
\hline & 33 & 0,324 & 52,6 & 25,21 & 0,11 & 0,060 \\
\hline & 49 & 0,369 & 46,5 & 30,32 & 0,13 & 0,083 \\
\hline & 67 & 0,757 & 50,5 & 74,40 & 0,26 & 0,232 \\
\hline & 73 & 0,531 & 40,2 & 48,72 & 0,17 & 0,101 \\
\hline & 83 & 0,388 & 45,4 & 32,48 & 0,13 & 0,078 \\
\hline & 94 & 0,385 & 53,2 & 32,14 & 0,15 & 0,115 \\
\hline & 98 & 0,380 & 48,8 & 31,57 & 0,14 & 0,064 \\
\hline & 114 & 0,841 & 35,6 & 83,94 & 0,21 & 0,354 \\
\hline & 132 & 1,118 & 49,2 & 115,40 & 0,31 & 0,488 \\
\hline & 145 & 0,558 & 40,8 & 51,79 & 0,18 & 0,174 \\
\hline & 157 & 0,820 & 49,8 & 81,55 & 0,27 & 0,189 \\
\hline \multirow[t]{12}{*}{ BR 101- PE } & 161 & 0,776 & 52,6 & 76,55 & 0,27 & 0,398 \\
\hline & 6 & 0,318 & 42,8 & 24,52 & 0,10 & 0,088 \\
\hline & 7 & 0,266 & 48,8 & 18,62 & 0,08 & 0,025 \\
\hline & 8 & 0,445 & 44,4 & 38,95 & 0,15 & 0,043 \\
\hline & 9 & 0,646 & 45,4 & 61,79 & 0,22 & 0,107 \\
\hline & 15 & 0,401 & 54,4 & 33,95 & 0,16 & 0,030 \\
\hline & 75 & 0,198 & 58,2 & 10,89 & 0,06 & 0,026 \\
\hline & 90 & 0,189 & 55,2 & 9,87 & 0,06 & 0,019 \\
\hline & 91 & 0,199 & 53,0 & 9,87 & 0,06 & 0,017 \\
\hline & 110 & 0,273 & 53,6 & 19,41 & 0,09 & 0,018 \\
\hline & 147 & 0,304 & 48,4 & 22,93 & 0,10 & 0,037 \\
\hline & 160 & 1,205 & 50,4 & 125,29 & 0,33 & 0,350 \\
\hline \multirow[t]{12}{*}{ BR 232 - PE } & 175 & 1,405 & 60,2 & 148,01 & 0,40 & 0,412 \\
\hline & $138 \mathrm{AC}$ & 0,385 & 50,0 & 32,14 & 0,14 & 0,062 \\
\hline & $138 \mathrm{C}$ & 0,299 & 50,2 & 22,37 & 0,10 & 0,029 \\
\hline & $138 \mathrm{DC}$ & 0,311 & 56,2 & 23,73 & 0,11 & 0,022 \\
\hline & $115 \mathrm{AC}$ & 0,345 & 58,2 & 27,59 & 0,13 & 0,041 \\
\hline & $115 \mathrm{C}$ & 0,207 & 60,4 & 11,92 & 0,06 & 0,043 \\
\hline & $115 \mathrm{DC}$ & 0,622 & 61,4 & 59,06 & 0,27 & 0,058 \\
\hline & $67 \mathrm{AC}$ & 0,388 & 48,2 & 32,48 & 0,14 & 0,084 \\
\hline & $67 \mathrm{C}$ & 0,509 & 58,8 & 46,22 & 0,22 & 0,095 \\
\hline & $67 \mathrm{DC}$ & 0,382 & 53,8 & 31,80 & 0,15 & 0,051 \\
\hline & $49 \mathrm{AC}$ & 0,249 & 49,0 & 16,69 & 0,08 & 0,033 \\
\hline & $49 \mathrm{C}$ & 0,589 & 51,2 & 55,31 & 0,22 & 0,099 \\
\hline BR 230 - PB & $49 \mathrm{DC}$ & 0,530 & 48,2 & 48,61 & 0,19 & 0,080 \\
\hline
\end{tabular}

Tabela 4. Resultados dos ensaios de mancha de areia das misturas simuladas em laboratório

\begin{tabular}{|c|c|c|c|c|c|c|c|}
\hline & \multicolumn{7}{|c|}{$H S(\mathrm{~mm})$} \\
\hline & Inicial & 100 ciclos & 300 ciclos & 1.000 ciclos & 3.000 ciclos & 10.000 ciclos & 30.000 ciclos \\
\hline Mistura D & 0,408 & 0,523 & 0,444 & 0,340 & 0,356 & 0,368 & 0,634 \\
\hline Mistura F & 0,503 & 0,525 & 0,503 & 0,662 & 0,617 & 0,446 & 0,765 \\
\hline
\end{tabular}

atrito.

Deve-se observar ainda que, as medidas de laboratório tendem a apresentar um valor superior àquelas encontradas em campo. Este fato pode ser explicado devido à forma como é feita a simulação, pois como o ensaio é realizado a $60^{\circ} \mathrm{C}$, o ligante que se desprende do agregado fica na roda de simulação e parte tende a voltar a cobrir o agregado. Por isto, as medidas de campo geralmente são inferiores às medidas encontradas em laboratório. Também deve-se salientar que, as medidas iniciais encontradas durante o ensaio com o pêndulo britânico sofrem influência da aderência da sapata com o ligante, destacando ainda mais que os valores de campo geralmente são inferiores àqueles encontrados em laboratório. Nota-se também que, em campo existem diversos agentes "polidores" e no laboratório somente o resíduo da areia que foi utilizada no ensaio de mancha de areia. Em campo existem ainda solicitações diferentes em função de vários fatores, tais como a geometria do local. Outro fator que foi observado é que a mistura com o maior TMN apresentou medidas de atrito superiores (Pereira, 2010).

Por fim, avaliando-se o IFI das misturas elaboradas em laboratório através da Tabela 5 e da Figura 5, verifica-se que a Mistura D apresentou valores abaixo do recomendado 


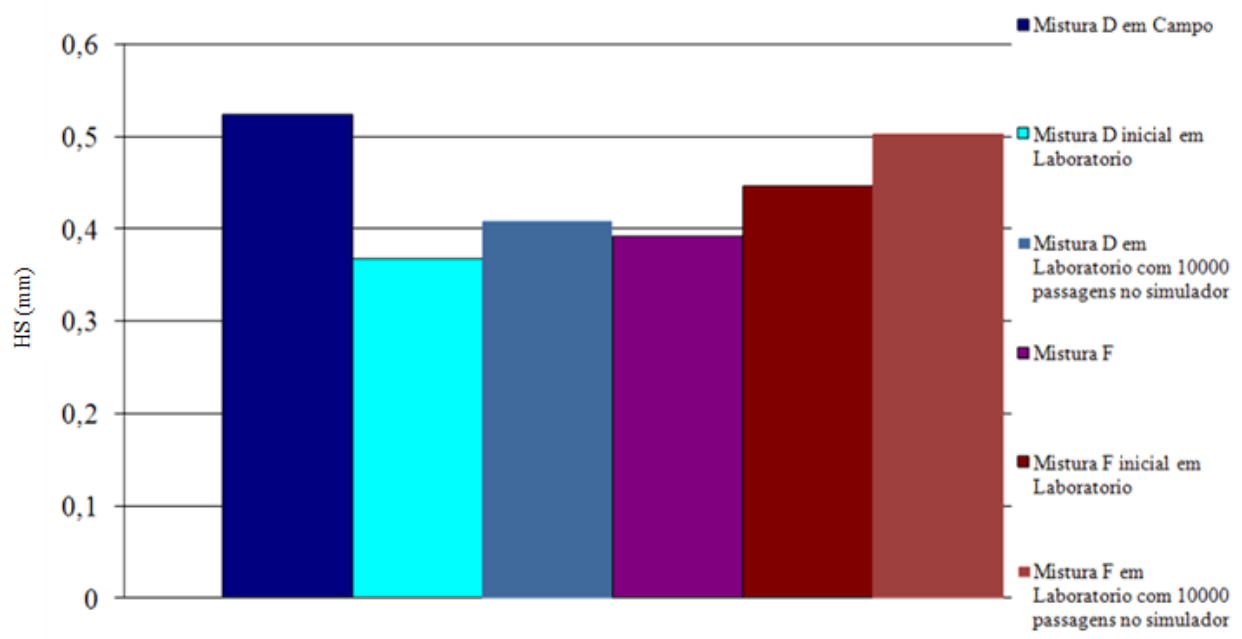

Figura 3. Comparação dos valores encontrados no ensaio de Mancha de Areia

Tabela 5. Resultados do ensaio de pêndulo britânico das misturas simuladas em laboratório

\begin{tabular}{lllll}
\hline & $\boldsymbol{B P N}$ & \multicolumn{3}{c}{ IFI (F60) } \\
\cline { 2 - 5 } & Inicial & 10.000 ciclos & Inicial & 10.000 ciclos \\
\hline Mistura D & 72,6 & 52,6 & 0,19 & 0,14 \\
Mistura F & 71,1 & 58,9 & 0,25 & 0,19 \\
\hline
\end{tabular}

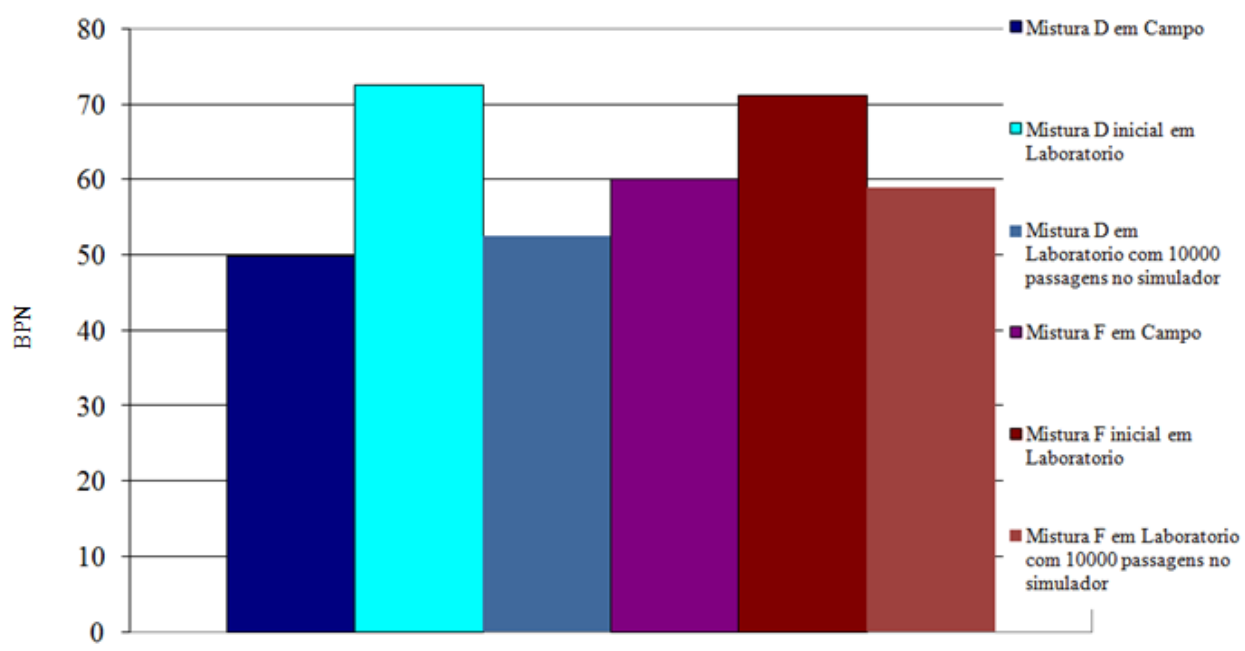

Figura 4. Comparação dos valores encontrados no ensaio de Pêndulo Britânico

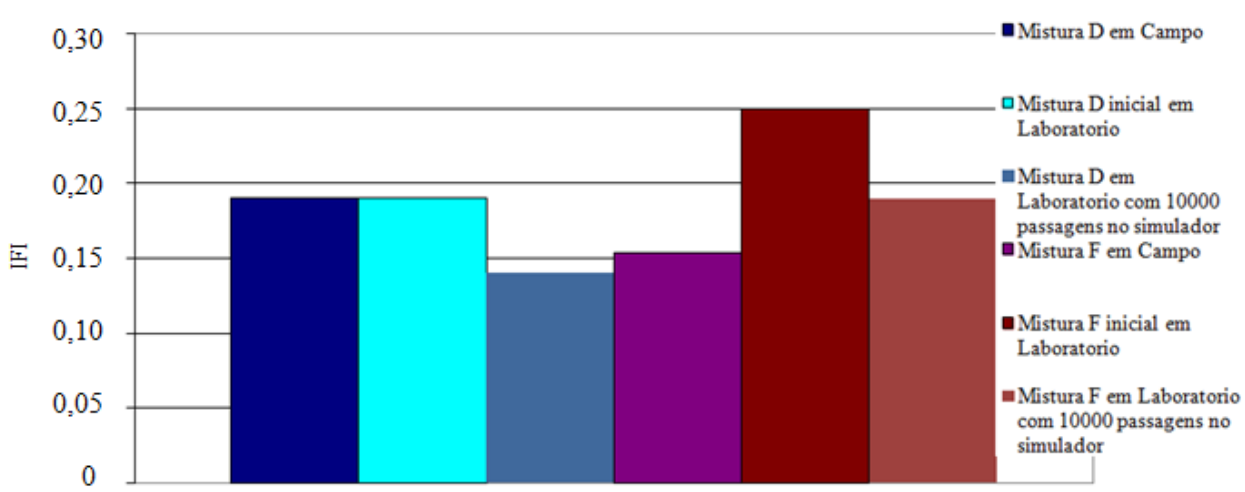

Figura 5. Comparação dos valores encontrados de IFI

para pavimentos em uso (correspondente aqui a 10.000 ciclos) e em estado inicial que são, respectivamente, 0,15 e 0,22 (Aps, 2010). Se os valores encontrados de IFI estão abaixo do recomendado é sinal de que o revestimento foi concebido de forma deficiente no que se refere à aderência pneu-pavimento (Pereira, 2010). Salienta ainda que, em laboratório foram observados valores superiores para a Mistura F e em campo a Mistura D apresentou melhor resultado. Isto pode ser explicado pelo fato das misturas apresentarem traços muito parecidos. Desta forma, sugerese que possam ser retirados apenas parâmetros mínimos de qualidade relativos a este aspecto em laboratório e não um 
ranqueamento entre misturas com graduação similar.

\subsection{Comparação dos resultados entre as misturas de campo simuladas em laboratório}

Elaboraram-se duas placas em laboratório, uma de CA e outra de SMA, utilizando as granulometrias apresentadas na Tabela 6. O teor de projeto de ligante para o CA e para o SMA foram, respectivamente, 5,4\% e 6,0\%. Primeiramente, foi realizada a caracterização das misturas abrangendo os ensaios de Módulo Resiliência (MR) (DNER ME 133-94), Resistência à Tração Indireta por Compressão Diametral (RT) (DNER ME 138-94) e Cântabro (DNER ME 383-99). Os resultados são apresentados na Tabela 7.

Em seguida, foram executados os ensaios de aderência pneu-pavimento nas placas moldadas na mesa compactadora do simulador de tráfego. Neste caso, foram feitas análises somente nas placas em estado inicial. Os ensaios de mancha de areia, pêndulo britânico e drenabilidade foram realizados nos dois lados da placa, sendo denominados $\mathrm{CA}(\mathrm{A})$ e $\mathrm{SMA}(\mathrm{A})$ o lado superior, ou seja, o lado onde houve contato com a roda da mesa compactadora, e $\mathrm{CA}(\mathrm{B})$ e $\mathrm{SMA}(\mathrm{B})$ o lado inferior, procurando não coincidir as áreas de realização dos ensaios. Os resultados dos ensaios podem ser observados na Tabela 8 .

Pode-se observar que, os resultados de mancha de areia para o CA ficaram abaixo do recomendado pelo DNIT (2006), que é de no mínimo 0,6mm. Os resultados encontrados podem ser usados para verificação da real adequação da mistura para regiões onde é requerida uma alta aderência entre pneu-pavimento, principalmente onde existe a possibilidade de ocorrer a formação de lâmina d'água. Este fato evidencia que a faixa $\mathrm{C}$ adotada para as misturas asfálticas utilizadas em grande parte das rodovias do país deve ser melhor analisada para que estas misturas não sejam concebidas de maneira inadequada perante o aspecto aderência pneu-pavimento. Comparando-se os valores obtidos no en- saio de mancha de areia para o SMA e para o CA, constatou-se que há um melhor desempenho à aderência no caso do SMA. Entretanto, convém mencionar que o SMA em geral é uma mistura mais onerosa do que o CA. Segundo Cavalcante (2005), na Paraíba um CA custa cerca de 78\% do valor de um SMA. Outro ponto observado é que, tanto em campo como em laboratório, houve alta correlação entre os valores de mancha de areia e de drenabilidade, evidenciando que a capacidade de drenar a água está diretamente relacionada à macrotextura do revestimento (Pereira, 2010).

Com relação às medidas de atrito, observou-se que, os valores encontrados em laboratório foram muito superiores aos encontrados em campo. Acredita-se que isto possa estar associado, principalmente, a não exposição da mistura ao tráfego. Pode-se pensar também que houve uma resistência entre a sapata do pêndulo e o ligante, ocasionando um aumento nos valores de atrito. Sabe-se que as medidas de atrito estão relacionadas à textura do agregado, como em laboratório a mistura não passou por nenhuma solicitação de modo a simular a ação do tráfego, esta ainda se encontra sem polimento por estar com os agregados recobertos pelo ligante asfáltico . Contudo, existem relatos de que o atrito ainda pode sofrer um acréscimo em relação às suas medidas iniciais, pois o pico do atrito se apresenta quando se tem o deslocamento da película de ligante e o agregado ainda se apresenta sem polimento. É importante verificar, ainda na fase de composição da mistura em laboratório, as características de textura em placas compactadas, antes de ensaios de trilha de roda. O polimento de agregados não é simples de ser verificado em laboratório em placas. Em geral, o polimento de agregados deve ser verificado por ensaio de polimento acelerado, que infelizmente ainda não existem no Brasil.

O IFI foi verificado para as duas placas confeccionadas em laboratório. Salvo um dos valores, foram encontrados bons resultados para o IFI, todos superiores a 0,22. Tam-

Tabela 6. Granulometrias (percentual passante em cada peneira)

\begin{tabular}{lll}
\hline & $\boldsymbol{C A}$ & $\boldsymbol{S M A}$ \\
\cline { 2 - 3 } Peneiras & $\%$ Passante & $\%$ Passante \\
\hline $3 / 4 "$ & 100 & 100 \\
$1 / 2 "$ & 93 & 95 \\
$3 / 8 "$ & 85 & 69 \\
$\mathrm{n}^{\circ} 4$ & 64 & 35 \\
$\mathrm{n}^{\circ} 10$ & 38 & 21 \\
$\mathrm{n}^{\circ} 40$ & 18 & 16 \\
$\mathrm{n}^{\circ} 80$ & 11 & 13 \\
$\mathrm{n}^{\circ} 200$ & 5 & 9 \\
\hline
\end{tabular}

Tabela 7. Resultados dos ensaios de caracterização da mistura

\begin{tabular}{lllll}
\hline Mistura & $\boldsymbol{M R}(\boldsymbol{M P a})$ & $\boldsymbol{R T}(\boldsymbol{M P a})$ & $\boldsymbol{M R} / \boldsymbol{R} \boldsymbol{T}$ & Cântabro (\%) \\
\hline CA & 2.832 & 0,88 & 3.224 & 4,1 \\
SMA & 3.196 & 0,88 & 3.613 & 3,8 \\
\hline
\end{tabular}

Tabela 8. Resultados dos ensaios para as misturas confeccionadas em laboratório

\begin{tabular}{lllll}
\hline & Mancha de & Pêndulo Britânico & & \\
Mistura & Areia $\boldsymbol{H S}(\mathbf{m m})$ & $\boldsymbol{B P N}$ & Drenabilidade $($ l/s) & IFI (F60) \\
\hline CA (A) & 0,509 & 83,6 & 0,016 & 0,28 \\
CA (B) & 0,301 & 81,0 & 0,008 & 0,13 \\
SMA (A) & 0,982 & 95,3 & 0,043 & 0,52 \\
SMA (B) & 0,918 & 95,3 & 0,028 & 0,50 \\
\hline
\end{tabular}


Tabela 9. Valores das correlações encontradas em campo e em laboratório

\begin{tabular}{|c|c|c|c|c|}
\hline \multirow[b]{2}{*}{ Correlação $R^{2}$} & \multicolumn{4}{|l|}{ Localidade } \\
\hline & Laboratório & $B R 101-P E$ & $B R 232-P E$ & $B R 230-P B$ \\
\hline $\mathrm{F} 60 \times \mathrm{Sp}$ & 0,99 & 0,90 & 0,98 & 0,96 \\
\hline $\mathrm{HS}(\mathrm{mm}) \times \mathrm{F} 60$ & 0,99 & 0,90 & 0,98 & 0,96 \\
\hline HS $(\mathrm{mm}) \times$ Vazão $(1 / \mathrm{s})$ & 0,89 & 0,85 & 0,96 & 0,54 \\
\hline F60 × Vazão (1/s) & 0,86 & 0,74 & 0,89 & 0,47 \\
\hline $\mathrm{BPN} \times \mathrm{F} 60$ & 0,96 & 0,01 & 0,03 & 0,04 \\
\hline
\end{tabular}

bém pode-se observar que os valores obtidos para a mistura do tipo SMA são superiores aos obtidos para o CA. Desta forma, os ensaios evidenciam que podem ser realizados ensaios para o ranqueamento entre misturas diferentes.

Verificando-se os padrões de comportamento das misturas em campo e em laboratório, como pode ser visto na Tabela 9, observa-se que existe um padrão entre as correlações, excetuando-se as relações com o pêndulo britânico. Em geral, as melhores correlações estão entre $\mathrm{HS}(\mathrm{mm})$ e F60, e em seguida a correlação entre F60 e Sp. Posteriormente, tem-se as correlações entre $\mathrm{HS}(\mathrm{mm})$ e vazão (1/s), e entre F60 e vazão(1/s), respectivamente. Estes resultados já eram esperados pois o F60 depende diretamente das medidas de textura e a vazão apresenta boa correlação com a mancha de areia, que, no caso deste trabalho, é apresentada como a medida de textura.

As correlações das medidas de atrito em campo não apresentaram o mesmo padrão do laboratório, o que pode ser explicado em função da dificuldade de simular em laboratório as medidas de atrito. Em campo tem-se o polimento do tráfego e, assim, as medidas dependem da resistência ao polimento e da forma dos agregados e, até mesmo, das características do tráfego.

\section{CONCLUSÕES}

Verificou-se neste trabalho que é difícil obter uma correlação direta entre as medidas de macrotextura obtidas em revestimentos asfálticos rodoviários após anos de operação e em laboratório com placas novas após ensaios de simulação de tráfego, pois existe um falso ganho nas medidas de textura em função das superfícies dos agregados ficarem mais salientes com o passar do pneu do simulador de tráfego. Porém, é possível verificar em laboratório, mesmo que não se reproduza exatamente a textura de campo, as tendências de macrotextura superficial em placas, antes de simulação. É possível também ranquear as misturas asfálticas em laboratório segundo a macrotextura obtida.

As medidas de atrito avaliadas em campo e em laboratório apresentaram boa correlação, contudo, os valores obtidos em laboratório apresentaram-se superiores aos obtidos em campo porque os agregados encontravam-se recobertos por ligante asfáltico. Assim, os ensaios de laboratório servem para uma previsão de comportamento, principalmente no que diz respeito às medidas iniciais, ou seja, pode-se verificar se o agregado possui um bom desempenho perante a microtextura, o que favorece o atrito entre o pneu e o pavimento. Também foi verificado que misturas que apresentam TMN superiores possuem um comportamento superior quanto ao atrito.

Analisando os valores de IFI verificou-se, para as misturas simuladas em laboratório, que uma das misturas apre- sentou resultados abaixo do sugerido por Aps (2010). Ressaltando ainda mais que se deve ter uma preocupação com a elaboração da mistura perante o aspecto aderência pneupavimento já na fase de concepção do projeto, pois se a região não apresentar disponibilidade de materiais que possa prover um bom atrito ao pavimento, a aderência deve ser compensada por uma mistura mais aberta para que se consiga atingir os valores mínimos sugeridos de IFI.

Verificou-se ainda que, o SMA apresentou-se superior ao $\mathrm{CA}$ no que se refere às medidas de aderência pneupavimento, o que já era esperado. Realizando-se os ensaios em laboratório comprovou-se que a análise serve para fazer um ranqueamento entre misturas, principalmente no caso das mesmas terem sido concebidas de maneira diferente. Neste sentido, pode-se realizar ainda na fase de projeto, uma classificação entre as misturas, baseadas nas características de macrotextura. Esta classificação pode ser obtida através de um banco de dados com parâmetros obtidos de diversos tipos de dosagens para a macrotextura, realizandose assim hierarquização entre elas. Desta forma, pode-se pensar em exigir uma faixa adequada de textura ainda na fase de projeto (Pereira, 2010).

Já as características de atrito, como foi mencionado anteriormente, estão mais ligadas às questões de rugosidade dos agregados. $\mathrm{O}$ estudo dos agregados de uma região pode fornecer um padrão de polimento em função das suas características mineralógicas. Desta forma, este fator também pode ser previsto ainda na fase de projeto realizando-se ensaios específicos nos agregados. Todos estes aspectos devem ser estudados na fase de concepção do projeto, principalmente em locais onde é exigida uma alta aderência entre pneu-pavimento. Com as informações aqui reportadas, espera-se contribuir para uma melhoria nas questões de aderência pneu-pavimento nas estradas, pois este aspecto representa ainda um fator de segurança viária pouco considerado no país.

\section{AGRADECIMENTOS}

Os autores agradecem ao Laboratório de Mecânica dos Pavimentos da Universidade Federal do Ceará (LMP/UFC) e ao Laboratório de Tecnologia de Pavimentação da Universidade de São Paulo (LTP/USP) pelo apoio. Ao Programa de Recursos Humanos da ANP (PRH26/ANP) pela bolsa de doutorado da primeira autora. Ao DNIT- PE e DNIT-PB pela disponibilização das informações. À Polícia Rodoviária Federal (PRF) de $\mathrm{PE}$ e da PB pela mobilização na realização dos ensaios de campo.

\section{REFERÊNCIAS BIBLIOGRÁFICAS}

Aps, M. (2006) Classificação da Aderência Pneu-Pavimento pelo Índice Combinado IFI - International Friction Index para Revestimentos Asfálticos. Tese (Doutorado), Escola Politécnica - USP, São Paulo,SP.

Aps, M. (2010) Análise da Aderência Pneu-Pavimento. Palestra proferida na Escola Politécnica - USP, São Paulo, SP em 14 de maio de 2010 . 
ASTM - American Society for Testing and Materials (1998) E 303-98 Standard Test Method for Measuring Surface Friction Properties Using the British Pendulum Tester.

ASTM - American Society for Testing and Materials (2001) E 965-01 Standard Test Method for Measuring Pavement Macrotexture Depth Using a Volumetric Technique.

ASTM - American Society for Testing and Materials (1998) E 1960 Standard Practice for Calculating International Friction Index of a Pavement Surface.

Bessa, I. S., V. T. F. Castelo Branco e J. B. SOARES (2011) Avaliação da Resistência ao Polimento e a Degradação de Agregados Utilizando a Abrasão Los Angeles e o Processamento Digital de Imagens. $7^{\circ}$ Congresso Brasileiro de Rodovias e Concessões, Anais..., Foz do Iguaçu.

Brosseaud, Y. (2006) Propriedade das Superficies dos Pavimentos em Relação à Segurança e ao Conforto dos Usuários. $18^{\circ}$ Encontro de Asfalto, Instituto Brasileiro de Petróleo e Gás - IBP, Rio de Janeiro, RJ.

Cavalcante, F. P. (2005) Avaliação das Características Funcionais e Estruturais da Rodovia BR - 230/PB Lote III. Dissertação (Mestrado), Universidade Federal de Pernambuco, Recife, PE.

CNT - Confederação Nacional do Transporte (2007) Pesquisa CNT de Rodovias 2007: Relatório Gerencial. CNT:SEST:SENAT. Brasília, DF.

CNT - Confederação Nacional do Transporte (2011) Pesquisa CNT de Rodovias 2011: Relatório Gerencial. CNT:SEST:SENAT. Brasília, DF.

DNER - Departamento Nacional de Estradas de Rodagem (1998). Manual de Reabilitação de Pavimentos Asfálticos. IPR, Rio de Janeiro, RJ.

DNIT - Departamento Nacional de Infraestrutura de Transportes (2006). Manual de Restauração de Pavimentos Asfálticos. IPR, Rio de Janeiro, RJ.

Ferreira, P. N. (2002) Avaliação da Macrotextura de Trechos Pavimentados de Rodovias Estaduais Situadas na Região Insular do Município de Florianópolis. Dissertação (Mestrado). Universidade Federal de Santa Catarina, Florianópolis, SC.

Lay, J. B. C. (1998) Friction and Surface Texture Characterization of 14 Pavement Test Sections in Greenville, North Carolina. Transportation Research Record, n. 1639, p.155-161. DOI: 10.3141/ 1639-17.

Mahmoud, E. M. (2005) Development of Experimental Methods for the Evaluation of Aggregate Resistance to Polishing, Abrasion and Breakage. Dissertação (Mestrado). Texas A\&M University, USA.

Masad, E., B. Muhunthan, N. Shashidhar e T. Harman (1999) Internal Structure Characterization of Asphalt Concrete Using Image Analysis. Journal of Computing in Civil Engineering, v. 13, n. 2 p. 88-95. DOI: 10.1061/(ASCE)0887-3801(1999)13:2(88).

Masad, E., T. Al-Rousan, J. Button, D. Little e E. Tutumluer (2005) Test Methods for Characterizing Aggregate Shape, Texture, and Angularity. NCHRP Project 4-30A, Texas Transportation Institute and University of Illinois at Urbana-Champaign.

Nunes, P. M., Y. Brousseaud, M. Do, P. Marsac, L. L. B. Bernucci e J. A. Quintanilha (2008) Characterization of in Laboratory and in Situ Macrotexture of a BBTM 06/ Mixture and Description of its Indicators. International Journal of Pavements, v. 7, p. 76-87.

Oliveira Filho, C. M. S. (2007) Estudo do Efeito de Diferentes Granulometrias no Comportamento Mecânico de Misturas Asfálticas Densas Descontínuas Tipo Stone Matrix Asphalt (SMA). Dissertação (Mestrado). Universidade Federal do Ceará, Fortaleza, CE.

Pereira, C. A. (2010) Análise da Aderência Pneu-Pavimento em Rodovias dos Estados de Pernambuco e da Paraiba com Elevado índice de Acidentes. Tese (Doutorado). Universidade Federal de Pernambuco, Recife, PE.

Pereira, C. A., J. B. Soares, I. D. S. Pontes Filho e V. T. F. Castelo Branco, (2011) Análise da Aderência Pneu-Pavimento em Pontos de Ocorrência de Acidentes. Revista Transportes, v. 20, n. 2, p. 6574. DOI:10.4237/transportes.v20i2.525.

Vasconcelos, K.L.; Evangelista, F. Jr.; Soares, J.B. (2005) Análise da Estrutura Interna de Misturas Asfálticas. XIX Congresso Nacional de Pesquisa e Ensino em Transportes, Anais..., ANPET, Recife, PE.

Yue, Z. Q., W. Bekking, I. Morin (1995) Application of Digital Image Processing to Quantitative Study of Asphalt Concrete Microstructure. Transportation Research Record, n. 1492, p. 53-60. 